\title{
Persistent yet reversible asymmetric pauciarthritis (PRAP): a B27-associated cluster
}

\author{
T. BItTER, M. JEANNET, E. De HALlER, AND M. LEJEUNE \\ From the Medical School, University of Lausanne, and the University Cantonal Hospital, Geneva, Switzerland
}

'Probable' rheumatoid arthritis (RA), as defined by the 1958 American Rheumatism Association $(\text { ARA })^{259}$ and the 1966 'New York' ${ }^{25}$ diagnostic criteria, includes several patients with a persistent yet non-erosive seronegative (rheumatoid factor negative) peripheral arthritis. ${ }^{336,225,211}$ In the face of more rigorous definitions of $\mathrm{RA}^{57,24}$ these cases remain 'unclassified' 108, 13, 234, 277 within the framework of presently accepted clinical syndromes and nomenclature. For lack of a more precise diagnosis they are variously referred to as 'seronegative rheumatoid arthritis', 'arthritis of unknown diagnosis', ${ }^{211,} 251$ or 'uncomplicated seronegative polyarthritis' ${ }^{336}$ Moreover, a considerable heterogeneity among patients with 'definite' and 'classical' RA has been emphasised by several authors. 251,247 This is further illustrated by a widely variable proportion of patients with HLA-B27 in various series. This association ranged from a near-control figure of $6 \cdot 1 \%{ }^{274}$ to over $30 \%$ in two series, ${ }^{62.163}$ presumably depending on the inclusion of borderline patients with a higher-than-normal degree of HLA-B27 association yet certainly distinct from Reiter's syndrome (RS).

Thus there is possibly a diagnostic gap, a hiatus, between established clinical entities. In fact, long before the discovery of HLA-B27 and its association with sacroiliitis and iritis-prone seronegative arthropathies ${ }^{61,276}$ asymmetry had been found to be of prognostic importance in RA and predictive of a benign course ${ }^{50,48}$ with spontaneous remission in $80 \%$ of cases of asymmetric seronegative polyarthritis ${ }^{287}$ temporarily set apart by Short and Bauer ${ }^{287,} 288$ as 'atypical' RA.

To discover whether the presumed diagnostic gap might be obscuring a single, thus far unrecognised, clinical entity 185 unselected consecutive patients with arthritis persisting for at least six weeks (that is, with three of the 1958 ARA diagnostic criteria for 'probable' RA) were entered in a five-year prospective clinical study. From 166 of these patients, available for a three-year follow-up study, cluster analysis and discriminant plots of the raw data yielded a discrete group of 40 presenting with a typical seronegative arthropathy, which we named PRAP after its four most discriminating (diagnostic) features: persistent yet reversible asymmetric pauciarthritis.

\section{Patients and methods}

From May 1972 to January 1975180 consecutive unselected outpatients of the Arthritis Clinic of Lausanne University Medical Centre were entered into a combined prospective and three-year followup study, provided they presented with a peripheral inflammatory arthropathy that had persisted for at least six weeks - that is, with at least three of the 1958 ARA and three out of four 'New York' criteria for the diagnosis of 'probable' rheumatoid arthritis. Exclusions, conventional clinical diagnoses, and the conventional age-16 cut-off for 'juvenile' polyarthritis were purposely ignored until after the cluster and discriminant analyses were finished in order not to introduce any bias into the computer evaluations central to the study.

The data presented here are limited to 166 patients available for clinical and radiological follow-up after at least 36 months. At entry and at follow-up each patient was asked for a history and given a complete physical examination with a careful search for bulbar conjunctivitis, mucous membrane lesions, balanitis, extensor tendonitis, plantar fasciitis and other enthesopathies, or heel pain. Cases of symptomatic cervicitis and adnexitis were confirmed by a gynaecologist. In men urethritis or a clear-cut history of it was accepted as evidence of 'genital inflammation'.

The pattern, extent, and intensity of inflammatory joint involvement was recorded on a manikin, each joint being scored for tenderness, hyperthermia, synovial thickening or effusion, or both (with superimposable symbols), yielding a modified Lansburytype index. ${ }^{216}$ The pattern was considered symmetric if at the first or follow-up examinations there were at least two pairs among five or more affected joints, or at least one pair among four or fewer inflamed 
joints. The pattern was termed asymmetric therefore when there was only one, or no pair among five or fewer affected joints and no pair among four or fewer affected joints. The degree of asymmetry was recorded quantitatively ${ }^{47}$ as the proportion of asymmetric joints and equalled:

$$
\frac{\text { Total No. of jts }-(\text { No. of pairs } \times 2)}{\text { Total No. of jts }}
$$

Special attention was paid to early morning low back pain and stiffness, typically relieved by movement, and to objective limitation of back movement recorded numerically using skin marks according to Schober as modified by Wright. ${ }^{228}$

The pattern and extent of joint and spine involvement as well as the erythrocyte sedimentation rate (Westergren) were recorded at intervals of 2 to 12 weeks and at a final follow-up visit at 36 months or later. This enabled the natural history of the arthropathy to be assessed.

At entry and at the final follow-up examination radiographs were taken of every affected joint as well as of the sacroiliac joints in every case in two views: (1) upright posteroanterior, and (2) anteroposterior with $15^{\circ}$ cephalad inclination. Tomograms were performed in doubtful cases. Radiological signs of periostitis as evidence of enthesopathy were looked for in lateral views of the calcaneum and in the radiographs of the clinically affected areas.

The rheumatoid factor titres by the latex test and by sheep cell agglutination (Professor K. Fehr, Kantonsspital, Zürich) were considered meaningful if positive at dilutions of $1: 80$ and $1: 16$, or higher, respectively. HLA was typed in every patient by one of us (M.J.) by the standard lymphocytotoxicity microtechnique ${ }^{226}$ with complete typing for $\mathrm{A}$ and
B loci in all pauciarticular cases and only B27 in the remainder.

\section{STATISTICAL ANALYSES}

The 166 patients completing the three-year followup were treated as an unselected continuum. Twenty-three clinical, radiological, and laboratory variables were selected ${ }^{107}$ for their potential weight in differentiating between subgroups of persistent, hence rheumatoid-like, ${ }^{259,} 25$ polyarthritides. They were used as a first step in a cluster analysis ${ }^{\mathbf{1 2 6}}$ and then, secondly, in a discriminant analysis. ${ }^{\mathbf{1 8 6}}$ The results, corroborated by multiple step-wise regressions (MSR), enabled the characteristics of patients within the PRAP cluster and those of all other patients with persistent arthritis to be compared. This yielded a relative weight for potential diagnostic criteria, given as discriminant function (respectively 'beta-elasticity' for MSR) coefficients. Moreover, discriminant analysis can allot each patient a relative position on a straight line which quantifies the degree of overlap, thus giving the probability of making a correct classification of a PRAP patient among others with equally persistent arthritis.

In a third step conventional diagnoses were introduced according to the definitions in Table 1 provided they were clear-cut and did not require additional qualifications such as 'definite', 'possible', or 'probable' after three years of follow-up. A discriminant plot then placed diagnosed patients (Fig. 1) and each cluster (Fig. 2) within a twodimensional area. The degree of heterogeneity of any group was assessed at cluster analysis of diagnosed patients by (1) the 'error' to be accepted in order to bring $90 \%$ or more of a given set of patients within

\begin{tabular}{|c|c|c|}
\hline Clinical characteristics & Disease & Source \\
\hline $\begin{array}{l}\text { Persistent, symmetrical erosive and/or frankly } \\
\text { seropositive polyarthritis in adults }\end{array}$ & $\mathbf{R A}$ & Present study, cluster analysis* \\
\hline $\begin{array}{l}\text { Asymmetric arthritis and/or tenosynovitis } \\
\text { with genital inflammation }\end{array}$ & RS & King, and Mason ${ }^{184}$ \\
\hline $\begin{array}{l}\text { Chronic asymmetric oligoarthritis in young } \\
\text { B27 }+ \text { males with two or more 'minor' }+ \\
\text { features of RS but no genital inflammation }\end{array}$ & Incomplete RS & Arnett ${ }^{17}, 18$ Jacobs $^{166}$ \\
\hline $\begin{array}{l}\text { Bilateral erosive or fusion sacroiliitis and B27 } \\
\text { phenotype (preceding or without skin or } \\
\text { gut involvement) }\end{array}$ & AS & Present study \\
\hline $\begin{array}{l}\text { Seronegative chronic arthritis and psoriasis } \\
\text { Mono-, pauci-, or polyarthritis starting below } \\
\text { age } 16\end{array}$ & $\begin{array}{l}\text { Psoriatic arthritis } \\
\text { Juvenile chronic arthritis }\end{array}$ & Wright and Moll ${ }^{332}$ \\
\hline
\end{tabular}

Table 1 Definition of clinical characteristics

*See discussion

+ 'Minor' features of RS: (1) definite periostitis, (2) calcaneal spurs and/or erosions, (3) plantar fasciitis, (4) extensor tenosynovitis, (5) diffuse 'sausage-like' swelling of isolated digits, (6) early morning low back pain (relieved by exercise), (7) rapid major weight loss, (8) acute onset, (9) mucuous membrane lesions or transient conjunctivitis without genital inflammation. 


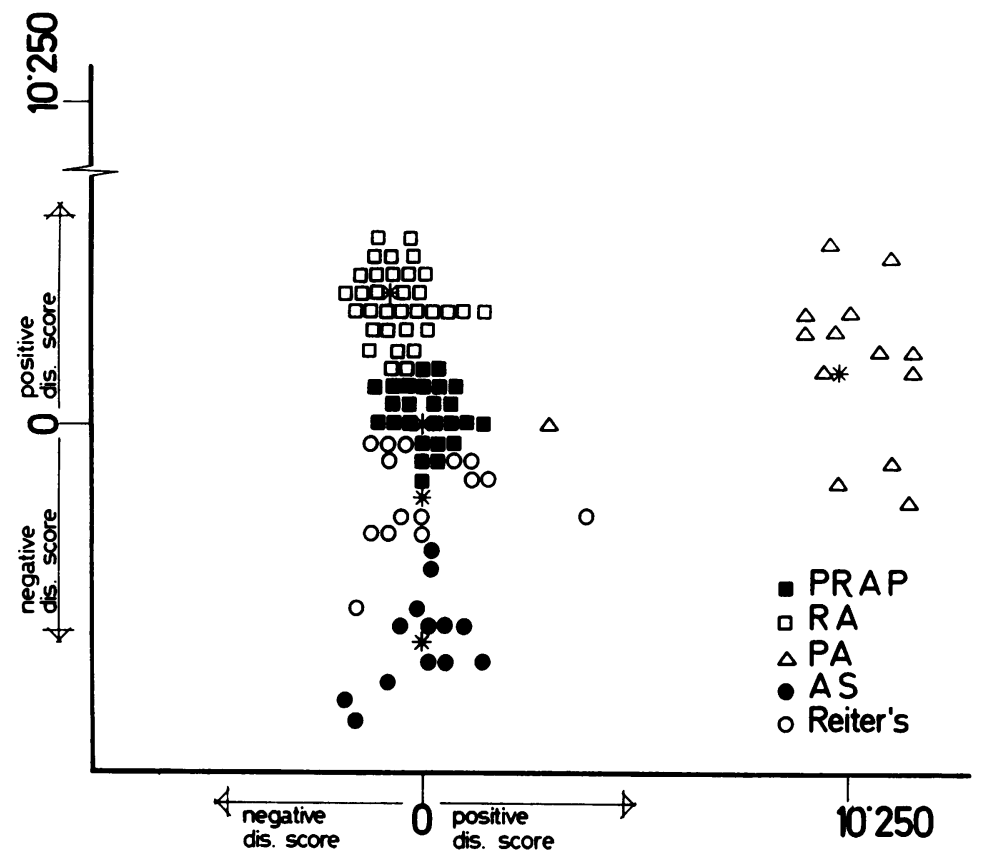

Fig. 1 Discriminant plot of individual patients with persistent arthritis as printed out by computer. Several patients of the same kind, when similarly placed, are represented by a single dot or symbol. Asterisks are centroids.

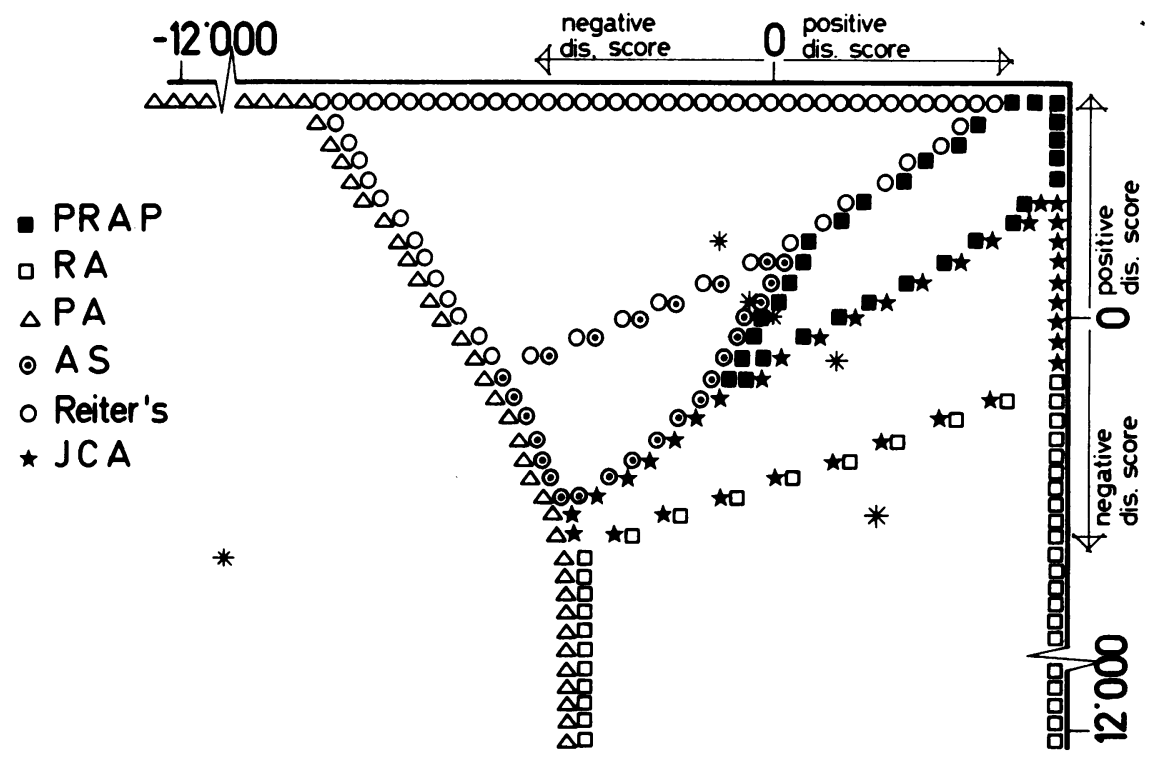

Fig. 2 Venn-type diagrammatic representation (computer-drawn discriminant plot) of 166 patients with six types of arthritis persisting over six weeks. Note PRAP located as wedge between Reiter's, AS, and JCA. Asterisks are centroids. 
a single cluster, and (2) the number of clustering steps thus required.

Eventually, in a fourth step, the clinical characteristics-that is, numerical or dichotomous variables-of patients with PRAP were compared with those seen in other established diseases in standard two-by-two analyses (Student's test for numerical variables: $\chi^{2}$ with Yates's correction as well as Fisher's exact test for dichotomous characteristics) with appropriate corrections. This also yielded the selectivity and specificity of dichotomous variables ${ }^{31}$ - that is, of potential diagnostic criteria.

\section{Results}

Among the 180 prospectively entered, unselected consecutive patients with arthritis persisting for over six weeks ${ }^{259} 166(89.7 \%)$ were available for a three-year follow-up study. Apart from eight groups of patients $(n=126)$ amenable to subsequent

Table 2 Articular and other characteristics (diagnostic criteria) of PRAP patients compared with controls ${ }^{1}$

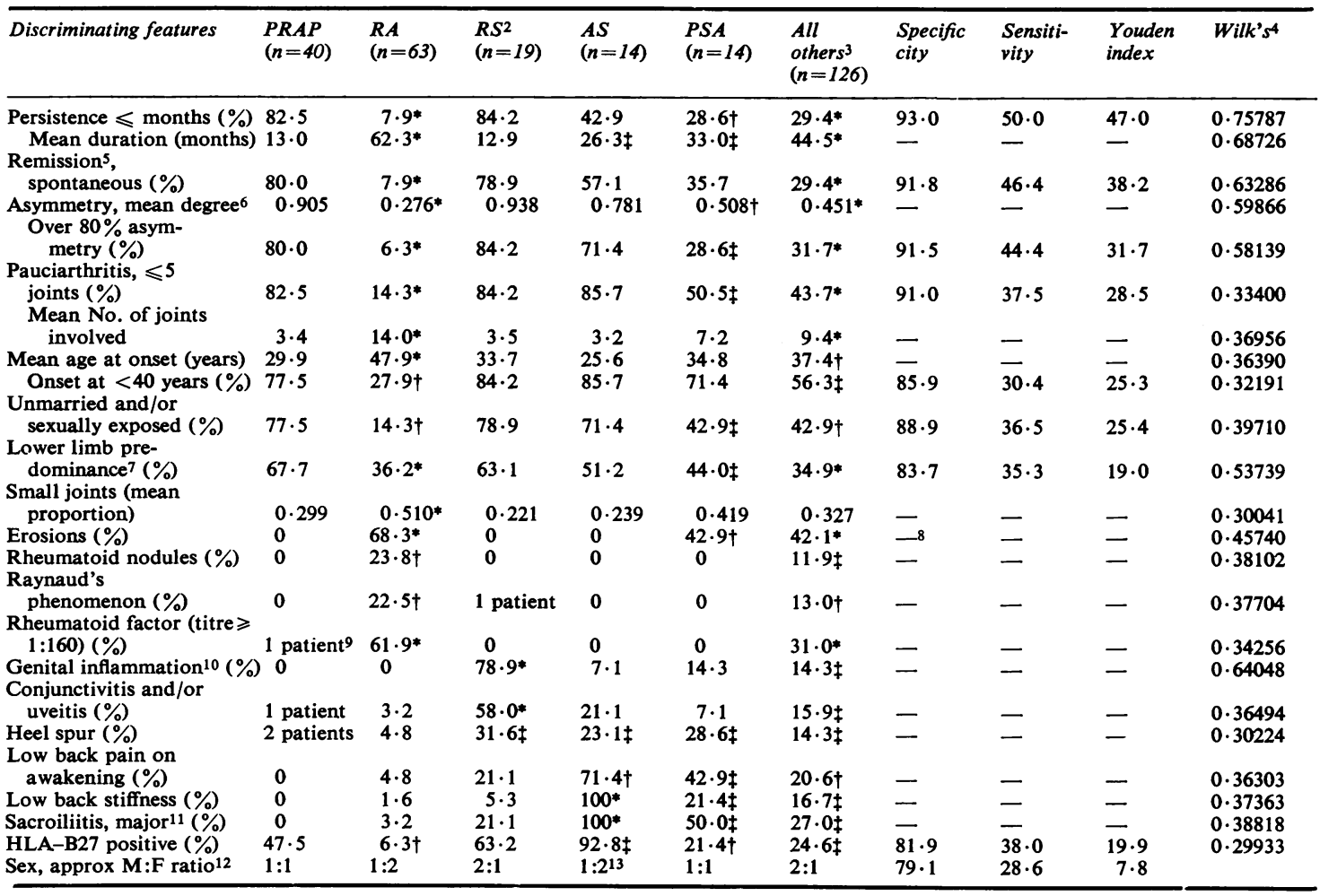

STATISTICAL SIGNIFICANCE

* $\mathrm{P}<0.0005$. $\dagger \mathrm{P}<0.005$. $¥ \mathrm{P}<0.05$. All others $=$ not significant.

1 Cumulated data and findings from three or more years of prospective observation. Continuous variables (means thereof) are expressed in decimal fractions of one and are not amenable to calculation of specificity, sensitivity, or Youden index. Dichotomous variables are shown in parentheses (\%).

2 Including incomplete RS, hence genital inflammation in only $78.9 \%$.

3 As controls all (non-PRAP) consecutive patients with arthritis persisting six or more weeks; tabulated as a pool to the right and by a diagnoses to the left of Table. Juvenile chronic arthritis $(n=16)$ has not been tabulated separately since it is too heterogeneous for valid comparisons.

4 Wilk's lambda, a discriminant coefficient, yields a relative 'weight' to potential (positive or negative) criteria.

5 Remission of peripheral arthritis and extra-articular manifestations, except sacroiliitis.

6 According to Bywaters ${ }^{37}$ : proportion of number of asymmetrically involved joints to total number of joints involved expressed as a decimal fraction of 1 .

7 A dichotomous variable: patients qualified only if there was $55 \%$ or more lower limb involvement.

8 Degrees of selectivity and specificity omitted in cases of negative Youden index.

9 One patient suffering from chronic aggressive hepatitis.

10 Urethritis and/or balanitis in men; adnexitis and/or cervicitis in women.

11 In a few B27-negative patients with normal sacroiliac joints on tomogram and on $99 \mathrm{mTc}$ scan.

12 Exact proportion of males used in statistical comparisons.

13 Reflects a female preponderance when elected for peripheral arthritis in the Lausanne series. ${ }^{168 a}$ 
conventional diagnoses ${ }^{328}$ cluster analysis yielded a discrete, highly homogeneous group of 40 young adults with a seronegative pauciarthritis. On a twodimensional discriminant plot these patients clustered between RA and the seronegative spondarthritides, in particular RS (Figs 1, 2).

The relative homogeneity within this cluster, seen on the discriminant plot as a great density of points (Fig. 1), may also be expressed as the reverse of the inherent 'error' on cluster analysis, which for PRAP $(0 \cdot 220)$ was alsmot twice that of RA $(0 \cdot 105)$. For a further check of homogeneity patients with PRAP were divided into two cohorts of B27positive $(n=19)$ and $B 27$-negative $(n=21)$ respectively. No significant difference could be found between those two subgroups.

The most discriminating features (Table 2) were (1) a short duration with early spontaneous remission in most when compared with other persistent arthritides, (2) a low degree of symmetry and often total asymmetry of joint involvement, (3) the small number of joints involved.

The twenty-three selected clinical, radiological, and laboratory characteristics recorded within this study were able to discriminate patients with PRAP from all others with a 'correct classification' in $90 \cdot 3 \%\left(X^{2}=107 ; P \leqslant 0.0001\right)$.
Indeed, PRAP differed from RS (Table 2) by lack of genital inflammation (urethritis and/or balanitis in men, adnexitis and/or cervicitis in women), by lack of early morning low back pain, by equal sex distribution, and a by lower degree of B27 association $(47.5 \%$ in PRAP). Moreover, chronicity and a number of 'minor features' (Table 1) of incomplete RS ${ }^{18,166}$ are conspicuously absent in PRAP.

After three years of follow-up none of the B27positive patients with PRAP had developed any low back pain or stiffness (so-called 'Rome criteria' ${ }^{25}$ for AS) or any erosive or bilateral radiological sacroiliitis. Minor (grade I, II) asymptomatic unilateral sacroiliitis was initially observed in only five B27negative patients and did not progress thereafter. They had no low back pain and no limitation of lumbar mobility.

Patients with PRAP differed significantly from RA in (1) a number of typically rheumatoid features (Table 2, lower half), including a different age-at-onset curve (Fig. 3); (2) a $47.5 \%$ B27association (as against $6.3 \%$ in RA); and (3) above all, by the benign non-deforming, nonerosive arthritis which lasted for less than 20 months in $82.5 \%$, having remitted thus far in 32 patients $(80 \cdot 0 \%)$.

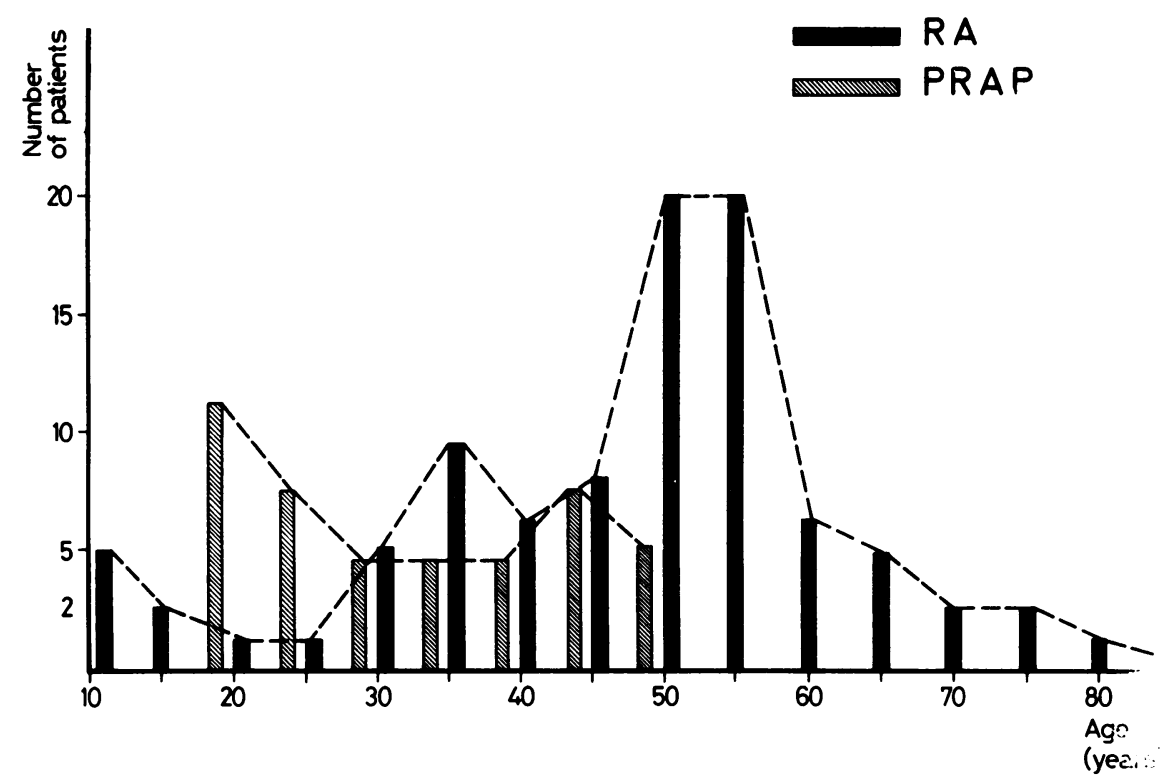

Fig. 3 Age at onset of rheumatoid arthritis $(R A)$ and persistent reversible asymmetric pauciarthritis $(P R A P)$. (Histogrammatic representation.) 


\section{Discussion}

Persistent yet reversible asymmetric pauciarthritis (PRAP), a discrete, homogeneous cluster of seronegative, non-erosive arthritides with a B27-association of $47.5 \%$, seems to fill a gap between RA and the seronegative spondarthritides, in particular RS. With three of the ARA and the so-called 'New York' diagnostic criteria for RA PRAP qualifies for 'probable' RA. It has actually accounted for $89.4 \%$ of all cases of 'atypical', 'possible', and 'probable' RA seen in Lausanne University Arthritis Clinic between 1972 and 1975.

A triple association of asymmetry, limited joint involvement, and a benign course despite initially persistent arthritis was specifically discussed by Bywaters in 1952 and $1961 .^{50,48}$ Benign polyarthritis ${ }^{200}$ as a seronegative asymmetric oligarthritis predominantly affecting the lower limbs, has been familiar to rheumatologists mostly under the name of 'atypical' RA coined by Short and Bauer. ${ }^{287,} 288$ Dixon $^{84}$ described two patients with asymmetric joint involvement as "both clinically and serologically atypical of rheumatoid arthritis, although no other diagnosis could be made on commonly accepted criteria.' Indeed, in a careful follow-up study of 212 (non-spondylitic) patients with RA Short and Bauer came across 17 such patients, 14 (82.3\%) of whom went into spontaneous complete remission as compared with $8 \%$ with PRAP. Short and Bauer hesitated to 'identify . . . an atypical form of RA'. But, before computer analysis was available for such follow-up data and without any genetic marker, they considered 'the clinical evidence insufficient for a rigid subdivision into ... typical and atypical forms of RA'-a subdivision which seems clearly warranted at this stage.

Except for the persistence of arthritis, however, RA differs from PRAP in just about every one of its typical features. A younger age at onset and a lack of female preponderance was specifically discussed by Short and Bauer in asymmetric reversible polyarthritis. Thus, what used to be called 'atypical RA' actually presents highly typical features of (1) asymmetry, (2) limited joint involvement, and (3) a good prognosis. A mild course and frequent complete remissions without recurrences sharply contrast with the eminently chronic course of Arnett's ${ }^{18}$ and Jacobs's ${ }^{166}$ 'incomplete Reiter's syndrome', also described by Joliat et al. ${ }^{169}$ under the title of 'atypical seronegative inflammatory arthropathy'. PRAP would seem to account for most of Masi's's1 patients with 'arthritis of unknown diagnosis', Feldmann's ${ }^{108}$ 'rhumatismes inclassifiables', and many cases of the 'rhumatisme subaigu curable de l'adulte' ${ }^{30}$ of the older French literature.

Despite an apparently high prevalence of PRAP it does not seem to be an early stage or a different form of AS, since none of the hallmarks of the latter developed within a three-year follow-up period. Characteristically the computer plots PRAP within an appreciable distance of AS both on individual plotting (Fig. 1) and the computer analogue of the Venn diagram (Fig. 2). Indeed, the peripheral arthritis of AS has been found significantly less asymmetric $(P<0.0001$, Table 2$)$ than that of PRAP. $A$ mild degree of unilateral non-erosive sacroiliitis, as seen in five patients with B27-negative pauciarthritis, is a regular feature of all the other 'seronegative spondarthritides' ${ }^{329}$, none of which is presently considered to be an alternate expression of AS.

Finally, we find ${ }^{168 a}$ that a therapeutic response within four days to high doses ( $\geqslant 600 \mathrm{mg} / 24 \mathrm{~h}$ ) of phenylbutazone* is almost diagnostic of AS, while the pain and inflammatory score of peripheral arthritis in PRAP, or, for that matter, of Milazzo's ${ }^{225}$ 'unclassified' seronegative patients, is remarkably resistant to such medication.

Future studies might show that persistent but ultimately reversible monarthritis in adults 110,227 might be to some extent B27-linked and belong to PRAP. The latter might well prove to be an extension into adulthood of persistent juvenile asymmetric rheumatism (and deserve the name of persistent adult reversible pauciarthritis).

\section{General discussion}

PROF. A. E. GOOD: This seems to be a very important paper because of the prevalence of this entity. It may become harder and harder to find healthy normal B27 carriers. What would be the approximate prevalence of PRAP compared with ankylosing spondylitis?

PROF. BITTER: This was not an epidemiological study, but in the Lausanne University Arthritis Clinic (where patients are self-referrals or sent by other specialists) the prevalence is three to five times that of RS and definitely as common as AS.

PROF. C. W. FINK: Such patients are probably very much more prevalent than actually seen in a rheumatology clinic. They are often thought to be of traumatic origin and treated by general practitioners or orthopaedists. Since they remit they are not sent to

*With serum phenylbutazone concentrations of $150 \mu \mathrm{g} / \mathrm{ml}$ or above 16 hours after the last intake. 
a rheumatologist. Childhood pauciarticular rheumatism also seems to be very common. Many children have one or a few joints affected for a few months, occasionally with recurrence a few years later.

PROF. BITTER: There is a retrospective questionnaire in a population study by Lawrence and Benett on 'benign polyarthritis'. They found bouts of spontaneously and completely remitting arthritis occurring in $5 \%$ of males and $10 \%$ of females in a population of 1234 .

DR. D. A. BREWERTON: Having been a bit caustic yesterday about the name 'incomplete Reiter's', I would like to underline the importance of this group of diseases. Heinrichs et al..$^{152}$ reported a very similar series in Helsinki three years ago. Milazzo ${ }^{225}$ from Adelaide reported another series which he called 'seronegative peripheral arthritis'. And then we heard yesterday from Dr Arnett about a similar group. All four reports seem to be referring to almost indentical groups of patients, although with different names.

DR. J. T. SCOTT: Just relevant to this is a study Fletcher and I did ${ }^{110}$ which was a retrospective follow-up of patients presenting with monarthritis of more than three months duration. I think there were about 150 cases. Twelve of them with more severe histological changes (on needle biopsy) went on to develop rheumatoid arthritis but the others ran a benign course. They settled down with no exacerbations. Have you histological information on these patients? PROF. BITTER: Yes, in one female patient there was mild non-specific synovitis without hypertrophy of the lining layers or any major lymphocyte infiltration beneath that.

DR. H. OTT: Did you find radiological abnormalities in your cases?

PROF. BITTER: We did not. The striking feature of this type of arthritis, in addition to the absence of rheumatoid factor, is non-erosiveness, which probably correlates with the spontaneous remission. This type of synovitis is very painful but $I$ am not sure whether it is sufficiently aggressive or hypertrophic to form a pannus. We haven't found a single eroded joint. I think that is the vital element in the diagnosis.

PROF. M. ZIFF: What has happened to these patients two years later? How long do they stay in remission?

PROF. BITTER: So far we have had only a three-year follow-up on them, but most of the patients have been in over two years of full remission by now. PROF. ZIFF: None of them have relapsed so far? PROF. BITTER: None.
PROF. R. F. WILLKENS: Have you typed these people for HBs antigen?

PROF. BITTER: No.

PROF. ZIFF: Did you find any clinical difference between B27-positive and B27-negative patients.

PROF. BITTER: We have split the PRAP patients into two groups and reanalysed them. We have found no single significant difference between B27-positive and B27-negative patients.

PROF. W. MARTEL: I think that is an important point to make. Judging from the literature I believe you should have found some radiological difference, particularly in view of B27-linked sacroiliitis.

PROF. BITTER: We did indeed find cases of mild, non-erosive unilateral sacroiliitis equally in both groups, but it introduced a wilful bias in that B27positive people with mild sacroiliitis were considered candidates for AS and excluded from PRAP. That left us with five B27-negative patients who, after three years of evolution, show mild unilateral sacroiliac involvement.

PROF. ZIFF: What is the average duration of arthritis in your PRAP patients?

PROF. BITTER: Thirteen months.

DR. F. C. ARNETT: This is a very interesting group indeed. I agree that they should be separated. Did you type them for any other B locus antigens, especially for B7, BW22, or BW42-that is, the B7 cross-reacting antigens (Cregs)?

PROF. BITTER: Yes, all pauciarticular patients got a complete HLA-A and HLA-B typing by Professor Jeannet, a co-author of this paper. However, B7 is a very common antigen in Western Europe, and I doubt very much whether one could find a significant difference in patients with PRAP.

DR. E. ALBERT: I don't quite understand your question about B7 Dr. Arnett. Do you think the incidence of B7 is raised in some forms of these diseases?

DR. ARNETT: Yes. In two-thirds of our RS patients who are negative for B27 we find one of the B7 Creg-either B7, BW22, or, in one of our black patients, BW42.

PROF. BITTER: This kind of observation would reach significance only if one introduced a correction factor for the number of alleles tested for.

DR. ALBERT: Yes, I think that calls for more sensitive tests in terms of significance. That is one thing. The second is that we tested this question in 220 patients and found no increased incidence of crossreacting antigens $\mathrm{B} 13, \mathrm{~B} 40$, and $\mathrm{BW} 22$. But $\mathrm{BW} 42$ we haven't tested.

DR. ARNETT: We did not find it in AS, only in RS and idiopathic sacroiliitis. Professor Bitter, do any of your PRAP patients have heel pain or sausage digits? 
PROF. BITTER: No sausage digits, a few had heel pain but no spur.

DR. ARNETT: I want to emphasise that I would not want anybody to think that my group 3 , which we call arthritis alone, was in fact only arthritis. It was arthritis but with other discriminating features. One of these is heel pain. How discriminating is heel pain for RS? Looking at Professor Wright's data on psoriatic arthritis the incidence of heel pain seems to be low in his series. In our series of nearly 40 patients with psoriatic arthritis heel pain seems to be prevalent only in those who have B27 as a bed fellow. In a previous study in the Air Force we did not find any patients with pure peripheral psoriatic arthritis who had heel pain. How high is the incidence of heel pain in ankylosing spondylitis? In our series it is only about $10 \%$, and in other diseases it is really quite rare. The sausage digit phenomenon in the absence of psoriasis may be very specific for RS. Similarly, how often does one find fever and weight loss in psoriatic arthritis, ankylosing spondylitis, or these other diseases?

PROF. BITTER: No fever in my series.

PROF. A. T. MASI: But seronegative polyarthritis is not necessarily asymmetric as the Table (Table 3 ) shows. PROF. BITTER: Such data rely heavily on the definition of RA. Your three asymmetrically involved patients with (presumably) seronegative, non-erosive RA would have clustered with PRAP, while, in our experience, symmetrical polyarthritis eventually (that is within three years) becomes erosive and/or seropositive-that is, definite or classical RA.
Table 3 Seronegative arthritis in males with younger adult onset (15-44 years) followed in arthritis research programme $(A R P)$

\begin{tabular}{llllll}
\hline $\begin{array}{l}\text { With or without } \\
\text { Bitter's symmetry } \\
\text { criteria }\end{array}$ & \multicolumn{2}{l}{ Clinical $R S$} & & \\
\cline { 2 - 5 } & Complete & Incomplete & Total & $R A$ & AUD \\
\hline With & 4 & 5 & 9 & 6 & 5 \\
Without & 2 & 5 & 7 & 3 & 9 \\
Total cases & 6 & 10 & 16 & 9 & 14 \\
$\quad$ Average age & & & $(23)$ & $(23)$ & $(31)$ \\
Black & & & 6 & 6 & 8 \\
White & & & 10 & 3 & 6 \\
\hline
\end{tabular}

DR J. C. WOODRow: I think that ultimately, when we think of aetiological considerations, somebody ought to go into the family histories of this group. What do you see in the first-degree relatives of these people compared with the patients with rheumatoid arthritis? You will find, I am sure, different genetic patterns.

PROF. BITTER: There were no consistent patterns in the family history. About $75 \%$ to $80 \%$ had no relevant family history, and in the others one could find any kind of rheumatic disease by history, in particular rheumatoid arthritis.

PROF. MARTEL: How complete was your radiological survey in these patients? Was it confined to affected joints or did you take a standard set of $x$-ray examinations on all patients, including the spine?

PROF. BITTER: We radiographed the affected joints, the heels, and the sacroiliac joints in every patient, both at onset and after a three-year follow-up. 